\title{
On the paranormed binomial sequence spaces
}

\author{
Hacer Bilgin Ellidokuzog̃lu ${ }^{\mathrm{a}}$, Serkan Demiriz ${ }^{\mathrm{b}^{*}}$ and Ali Köseog̃lu ${ }^{\mathrm{a}}$ \\ ${ }^{a}$ Recep Tayyip Erdogan University, Science and Art Faculty, Department of Mathematics, Rize-Turkey \\ ${ }^{\mathrm{b}}$ Gaziosmanpaşa University, Science and Art Faculty, Department of Mathematics, Tokat-Turkey \\ ${ }^{*}$ Corresponding author E-mail: serkandemiriz@gmail.com
}

\section{Article Info}

Keywords: Binomial sequence spaces, Paranorm, Matrix domain, Matrix transformations

2010 AMS: 46A45, 40C05, 46B20

Received: 15 February 2018

Accepted: 6 March 2018

Available online: 30 September 2018

\begin{abstract}
In this paper the sequence spaces $b_{0}^{r, s}(p), b_{c}^{r, s}(p), b_{\infty}^{r, s}(p)$ and $b^{r, s}(p)$ which are the generalization of the classical Maddox's paranormed sequence spaces have been introduced and proved that the spaces $b_{0}^{r, s}(p), b_{c}^{r, s}(p), b_{\infty}^{r, s}(p)$ and $b^{r, s}(p)$ are linearly isomorphic to spaces $c_{0}(p), c(p), \ell_{\infty}(p)$ and $\ell(p)$, respectively. Besides this, the $\alpha-, \beta-$ and $\gamma-$ duals of the spaces $b_{0}^{r, s}(p), b_{c}^{r, s}(p)$, and $b^{r, s}(p)$ have been computed, their bases have been constructed and some topological properties of these spaces have been studied. Finally, the classes of matrices $\left(b_{0}^{r, s}(p): \mu\right),\left(b_{c}^{r, s}(p): \mu\right)$ and $\left(b^{r, s}(p): \mu\right)$ have been characterized, where $\mu$ is one of the sequence spaces $\ell_{\infty}, c$ and $c_{0}$ and derives the other characterizations for the special cases of $\mu$.
\end{abstract}

\section{Introduction}

We shall denote the space of all real-valued sequences by $w$ as a classical notation. Any vector subspace of $w$ is called a sequence space. The spaces $\ell_{\infty}, c$ and $c_{0}$ are the most common and frequently used spaces which are all bounded, convergent and null sequences, respectively. Also $b s, c s, \ell_{1}$ and $\ell_{p}$ notations are used for the spaces of all bounded, convergent, absolutely and $p$-absolutely convergent series, respectively, where $1<p<\infty$.

First, we point out the concept of a paranorm. A linear topological space $X$ over the real field $\mathbb{R}$ is said to be a paranormed space if there is a subadditive function $g: X \rightarrow \mathbb{R}$ such that $g(\theta)=0, g(x)=g(-x)$ and scalar multiplication is continuous, i.e., $\left|\alpha_{n}-\alpha\right| \rightarrow 0$ and $g\left(x_{n}-x\right) \rightarrow 0$ imply $g\left(\alpha_{n} x_{n}-\alpha x\right) \rightarrow 0$ for all $\alpha$ 's in $\mathbb{R}$ and all $x$ 's in $X$, where $\theta$ is the zero vector in the linear space $X$.

Assume here and after that $\left(p_{k}\right)$ be a bounded sequences of strictly positive real numbers with $\sup p_{k}=H$ and $L=\max \{1, H\}$. Then, the linear spaces $\ell_{\infty}(p), c(p), c_{0}(p)$ and $\ell(p)$ were defined by Maddox [19] (see also Simons [21] and Nakano [20]) as follows:

$$
\begin{aligned}
& \ell_{\infty}(p)=\left\{x=\left(x_{k}\right) \in w: \sup _{k \in \mathbb{N}}\left|x_{k}\right|^{p_{k}}<\infty\right\}, \\
& c(p)=\left\{x=\left(x_{k}\right) \in w: \lim _{k \rightarrow \infty}\left|x_{k}-l\right|^{p_{k}}=0 \text { for some } l \in \mathbb{R}\right\}, \\
& c_{0}(p)=\left\{x=\left(x_{k}\right) \in w: \lim _{k \rightarrow \infty}\left|x_{k}\right|^{p_{k}}=0\right\} \\
& \ell(p)=\left\{x=\left(x_{k}\right) \in w: \sum_{k}\left|x_{k}\right|^{p_{k}}<\infty\right\},
\end{aligned}
$$

which are the complete spaces paranormed by

$$
g_{1}(x)=\sup _{k \in \mathbb{N}}\left|x_{k}\right|^{p_{k} / L} \Longleftrightarrow \inf p_{k}>0 \text { and } g_{2}(x)=\left(\sum_{k}\left|x_{k}\right|^{p_{k}}\right)^{1 / L},
$$

respectively. For convenience in notation, here and in what follows, the summation without limits runs from 0 to $\infty$. By $\mathscr{F}$ and $\mathbb{N}_{k}$, we shall denote the collection of all finite subsets of $\mathbb{N}$ and the set of all $n \in \mathbb{N}$ such that $n \geq k$, respectively. We shall assume throughout that $p_{k}^{-1}+\left(p_{k}^{\prime}\right)^{-1}=1$ provided $1<\inf p_{k} \leq H<\infty$. 
Let $\lambda, \mu$ be any two sequence spaces and $A=\left(a_{n k}\right)$ be an infinite matrix of real numbers $a_{n k}$, where $n, k \in \mathbb{N}$. Then, we say that $A$ defines a matrix mapping from $\lambda$ into $\mu$, and we denote it by $A: \lambda \rightarrow \mu$, if for every sequence $x=\left(x_{k}\right) \in \lambda$, the sequence $A x=\left\{(A x)_{n}\right\}$, the $A$-transform of $x$, is in $\mu$, where

$$
(A x)_{n}=\sum_{k} a_{n k} x_{k},(n \in \mathbb{N})
$$

By $(\lambda: \mu)$, we denote the class of all matrices $A$ such that $A: \lambda \rightarrow \mu$. Thus, $A \in(\lambda: \mu)$ if and only if the series on the right-hand side of (1.1) converges for each $n \in \mathbb{N}$ and every $x \in \lambda$, and we have $A x=\left\{(A x)_{n}\right\}_{n \in \mathbb{N}} \in \mu$ for all $x \in \mu$. A sequence $x$ is said to be $A$-summable to $\alpha$ if $A x$ converges to $\alpha$ which is called the $A$-limit of $x$.

\section{The sequence spaces $b_{0}^{r, s}(p), b_{c}^{r, s}(p), b_{\infty}^{r, s}(p)$ and $b^{r, s}(p)$}

In this section, we define the sequence spaces $b_{0}^{r, s}(p), b_{c}^{r, s}(p), b_{\infty}^{r, s}(p)$ and $b^{r, s}(p)$, and prove that $b_{0}^{r, s}(p), b_{c}^{r, s}(p), b_{\infty}^{r, s}(p)$ and $b^{r, s}(p)$ are the complete paranormed linear spaces.

For a sequence space $\lambda$, the matrix domain $\lambda_{A}$ of an infinite matrix $A$ is defined by

$$
\lambda_{A}=\left\{x=\left(x_{k}\right) \in w: A x \in \lambda\right\} .
$$

In [7], Choudhary and Mishra have defined the sequence space $\overline{\ell(p)}$ which consists of all sequences such that $S-\operatorname{transforms~are~in~} \ell_{(p)}$, where $S=\left(s_{n k}\right)$ is defined by

$$
s_{n k}=\left\{\begin{array}{cc}
1 & , \quad 0 \leq k \leq n \\
0 & , \quad k>n
\end{array}\right.
$$

Başar and Altay [3] have studied the space $b s(p)$ which is formerly defined by Başar in [4] as the set of all series whose sequences of partial sums are in $\ell_{\infty}(p)$. More recently, Altay and Başar have studied the sequence spaces $r^{t}(p), r_{\infty}^{t}(p)$ in [1] and $r_{c}^{t}(p), r_{0}^{t}(p)$ in [2] which are derived by the Riesz means from the sequence spaces $\ell(p), \ell_{\infty}(p), c(p)$ and $c_{0}(p)$ of Maddox, respectively.

With the notation of (2.1), the spaces $\overline{\ell(p)}, b s(p), r^{t}(p), r_{\infty}^{t}(p), r_{c}^{t}(p)$ and $r_{0}^{t}(p)$ may be redefined by

$$
\begin{aligned}
& \overline{\ell(p)}=[\ell(p)]_{S}, b s(p)=\left[\ell_{\infty}(p)\right]_{S}, r^{t}(p)=[\ell(p)]_{R}^{t} \\
& r_{\infty}^{t}(p)=\left[\ell_{\infty}(p)\right]_{R}^{t}, r_{c}^{t}(p)=[c(p)]_{R}^{t}, r_{0}^{t}(p)=\left[c_{0}(p)\right]_{R}^{t} .
\end{aligned}
$$

In [8], Demiriz and Çakan have defined the sequence spaces $e_{0}^{r}(u, p)$ and $e_{c}^{r}(u, p)$ which consists of all sequences such that $E^{r, u}$ - transforms are in $c_{0}(p)$ and $c(p)$, respectively $E^{r, u}=\left\{e_{n k}^{r}(u)\right\}$ is defined by

$$
e_{n k}^{r}(u)=\left\{\begin{array}{ccc}
\left(\begin{array}{l}
n \\
k
\end{array}\right)(1-r)^{n-k} r^{k} u_{k} & , \quad(0 \leq k \leq n), \\
0 & , \quad(k>n)
\end{array}\right.
$$

for all $k, n \in \mathbb{N}$ and $0<r<1$.

In [5] and [6], the Binomial sequence spaces $b_{0}^{r, s}, b_{c}^{r, s}, b_{\infty}^{r, s}$ and $b_{p}^{r, s}$, which are the matrix domains of Binomial mean $B^{r, s}$ in the sequence spaces $c_{0}, c, \ell_{\infty}$ and $\ell_{p}$, respectively, are introduced, some inclusion relations and Schauder basis for the spaces $b_{0}^{r, s}, b_{c}^{r, s}, b_{\infty}^{r, s}$ and $b_{p}^{r, s}$ are given, and the $\alpha-, \beta-$ and $\gamma-$ duals of those spaces are determined. For more papers related to sequence spaces and matrix domains of different infinite matrices one can see $[13,12]$ and references therein. The main purpose of this paper is to introduce the sequence spaces $b_{0}^{r, s}(p), b_{c}^{r, s}(p), b_{\infty}^{r, s}(p)$ and $b^{r, s}(p)$ which are the set of all sequences whose $B^{r, s}$-transforms are in the spaces $c_{0}(p), c(p), \ell_{\infty}(p)$ and $\ell(p)$, respectively; where $B^{r, s}$ denotes the matrix $B^{r, s}=\left\{b_{n k}^{r, s}\right\}$ defined by

$$
b_{n k}^{r, s}=\left\{\begin{array}{ccc}
\frac{1}{(s+r)^{n}}\left(\begin{array}{l}
k \\
n
\end{array}\right) s^{n-k} r^{k} & , & 0 \leq k \leq n, \\
0 & , & k>n,
\end{array}\right.
$$

where $s r>0$. Also, we have constructed the basis and computed the $\alpha-, \beta-$ and $\gamma$-duals and investigated some topological properties of the spaces $b_{0}^{r, s}(p), b_{c}^{r, s}(p), b_{\infty}^{r, s}(p)$ and $b^{r, s}(p)$.

Following Choudhary and Mishra [7], Başar and Altay [3], Altay and Başar [1, 2], Demiriz [8], Kirişçi [14, 15], Candan and Güneş [16] and Ellidokuzog̃lu and Demiriz [9], we define the sequence spaces $b_{0}^{r, s}(p), b_{c}^{r, s}(p), b_{\infty}^{r, s}(p)$ and $b^{r, s}(p)$, as the sets of all sequences such that $B^{r, s}$-transforms of them are in the spaces $c_{0}(p), c(p), \ell_{\infty}(p)$ and $\ell(p)$, respectively, that is,

$$
\begin{aligned}
& b_{0}^{r, s}(p)=\left\{x=\left(x_{k}\right) \in w: \lim _{n \rightarrow \infty}\left|\frac{1}{(s+r)^{n}} \sum_{k=0}^{n}\left(\begin{array}{l}
n \\
k
\end{array}\right) s^{n-k} r^{k} x_{k}\right|^{p_{n}}=0\right\}, \\
& b_{c}^{r, s}(p)=\left\{x=\left(x_{k}\right) \in w: \exists l \in \mathbb{C} \ni \lim _{n \rightarrow \infty}\left|\frac{1}{(s+r)^{n}} \sum_{k=0}^{n}\left(\begin{array}{l}
n \\
k
\end{array}\right) s^{n-k} r^{k} x_{k}-l\right|^{p_{n}}=0\right\}, \\
& b_{\infty}^{r, s}(p)=\left\{x=\left(x_{k}\right) \in w: \sup _{n \in \mathbb{N}}\left|\frac{1}{(s+r)^{n}} \sum_{k=0}^{n}\left(\begin{array}{l}
n \\
k
\end{array}\right) s^{n-k} r^{k} x_{k}\right|^{p_{n}}<\infty\right\}, \\
& b^{r, s}(p)=\left\{x=\left(x_{k}\right) \in w: \sum_{n}\left|\frac{1}{(s+r)^{n}} \sum_{k=0}^{n}\left(\begin{array}{l}
n \\
k
\end{array}\right) s^{n-k} r^{k} x_{k}\right|^{p_{n}}<\infty\right\} .
\end{aligned}
$$


In the case $\left(p_{n}\right)=e=(1,1,1, \ldots)$, the sequence spaces $b_{0}^{r, s}(p), b_{c}^{r, s}(p), b_{\infty}^{r, s}(p)$ and $b^{r, s}(p)$ are, respectively, reduced to the sequence spaces $b_{0}^{r, s}, b_{c}^{r, s}, b_{\infty}^{r, s}$ and $b_{p}^{r, s}$ which are introduced by Bişgin [5, 6]. With the notation of $(2.1)$, we may redefine the spaces $b_{0}^{r, s}(p), b_{c}^{r, s}(p), b_{\infty}^{r, s}(p)$ and $b^{r, s}(p)$ as follows:

$$
b_{0}^{r, s}(p)=\left[c_{0}(p)\right]_{B^{r, s}}, b_{c}^{r, s}(p)=[c(p)]_{B^{r, s}}, b_{\infty}^{r, s}(p)=\left[\ell_{\infty}(p)\right]_{B^{r, s}} \text { and } b^{r, s}(p)=[\ell(p)]_{B^{r, s}}
$$

Define the sequence $y=\left\{y_{n}(r, s)\right\}$, which will be frequently used, as the $B^{r, s}-$ transform of a sequence $x=\left(x_{k}\right)$, i.e.,

$$
y_{n}(r, s):=\frac{1}{(s+r)^{n}} \sum_{k=0}^{n}\left(\begin{array}{l}
n \\
k
\end{array}\right) s^{n-k} r^{k} x_{k} ; \text { for all } k \in \mathbb{N} .
$$

Now, we may begin with the following theorem which is essential in the text.

Theorem 2.1. $b_{0}^{r, s}(p), b_{c}^{r, s}(p)$ and $b_{\infty}^{r, s}(p)$ are the complete linear metric space paranormed by $g$, defined by

$$
g(x)=\sup _{n \in \mathbb{N}}\left|\frac{1}{(s+r)^{n}} \sum_{k=0}^{n}\left(\begin{array}{l}
n \\
k
\end{array}\right) s^{n-k} r^{k} x_{k}\right|^{p_{n} / L} .
$$

In addition, $b^{r, s}(p)$ is the complete linear metric space paranormed by $h$, defined by

$$
h(x)=\left(\sum_{n=0}^{\infty}\left|\frac{1}{(s+r)^{n}} \sum_{k=0}^{n}\left(\begin{array}{l}
n \\
k
\end{array}\right) s^{n-k} r^{k} x_{k}\right|^{p_{n}}\right)^{1 / M} .
$$

Proof. First, we give the proof for $b_{0}^{r, s}(p), b_{c}^{r, s}(p)$ and $b_{\infty}^{r, s}(p)$. Since the proof is similar for $b_{c}^{r, s}(p)$ and $b_{\infty}^{r, s}(p)$, we give the proof only for the space $b_{0}^{r, s}(p)$. The linearity of $b_{0}^{r, s}(p)$ with respect to the co-ordinatewise addition and scalar multiplication follows from the following inequalities which are satisfied for $x, z \in b_{0}^{r, s}(p)$ (see Maddox [18, p.30])

$$
\left|\frac{1}{(s+r)^{n}} \sum_{k=0}^{n}\left(\begin{array}{l}
n \\
k
\end{array}\right) s^{n-k} r^{k}\left(x_{k}+z_{k}\right)\right|^{p_{n} / L} \leq\left|\frac{1}{(s+r)^{n}} \sum_{k=0}^{n}\left(\begin{array}{l}
n \\
k
\end{array}\right) s^{n-k} r^{k} x_{k}\right|^{p_{n} / L}+\left|\frac{1}{(s+r)^{n}} \sum_{k=0}^{n}\left(\begin{array}{l}
n \\
k
\end{array}\right) s^{n-k} r^{k} z_{k}\right|^{p_{n} / L}
$$

and for any $\alpha \in \mathbb{R}$ (see [21])

$$
|\alpha|^{p_{n}} \leq \max \left\{1,|\alpha|^{L}\right\}=K
$$

Using (2.6) inequality, we get

$$
\begin{aligned}
\left|\frac{1}{(s+r)^{n}} \sum_{k=0}^{n}\left(\begin{array}{l}
n \\
k
\end{array}\right) s^{n-k} r^{k}\left(\alpha x_{k}\right)\right|^{p_{n} / L} & =|\alpha|^{p_{n} / L}\left|\frac{1}{(s+r)^{n}} \sum_{k=0}^{n}\left(\begin{array}{l}
n \\
k
\end{array}\right) s^{n-k} r^{k} x_{k}\right|^{p_{n} / L} \\
& \leq K^{1 / L}\left|\frac{1}{(s+r)^{n}} \sum_{k=0}^{n}\left(\begin{array}{l}
n \\
k
\end{array}\right) s^{n-k} r^{k} x_{k}\right|^{p_{n} / L}
\end{aligned}
$$

for $x \in b_{0}^{r, s}(p)$. This shows the space $b_{0}^{r, s}(p)$ is a linear space.

Now we will see that $g$ is a paranorm on $b_{0}^{r, s}(p)$. It is clear that $g(\theta)=0$ and $g(x)=g(-x)$ for all $x \in b_{0}^{r, s}(p)$.

Let $\left\{x^{n}\right\}$ be any sequence of the points $x^{n} \in b_{0}^{r, s}(p)$ such that $g\left(x^{n}-x\right) \rightarrow 0$ and $\left(\alpha_{n}\right)$ also be any sequence of scalars such that $\alpha_{n} \rightarrow \alpha$. Then, since the inequality

$$
g\left(x^{n}\right) \leq g(x)+g\left(x^{n}-x\right)
$$

holds by the subadditivity of $g,\left\{g\left(x^{n}\right)\right\}$ is bounded and we thus have

$$
\begin{aligned}
g\left(\alpha_{n} x^{n}-\alpha x\right) & =\sup _{k \in \mathbb{N}}\left|\frac{1}{(s+r)^{k}} \sum_{j=0}^{k}\left(\begin{array}{c}
k \\
j
\end{array}\right) s^{k-j} r^{j}\left(\alpha_{n} x_{j}^{n}-\alpha x_{j}\right)\right|^{p_{k} / L} \\
& \leq\left|\alpha_{n}-\alpha\right| g\left(x^{n}\right)+|\alpha| g\left(x^{n}-x\right)
\end{aligned}
$$

which tends to zero as $n \rightarrow \infty$. This means that the scalar multiplication is continuous. Hence, $g$ is a paranorm on the space $b_{0}^{r, s}(p)$.

It remains to prove the completeness of the space $b_{0}^{r, s}(p)$. Let $\left\{x^{i}\right\}$ be any Cauchy sequence in the space $b_{0}^{r, s}(p)$, where $x^{i}=\left\{x_{0}^{(i)}, x_{1}^{(i)}, x_{2}^{(i)}, \ldots\right\}$. Then, for a given $\varepsilon>0$ there exists a positive integer $n_{0}(\varepsilon)$ such that

$$
g\left(x^{i}-x^{j}\right)<\frac{\varepsilon}{2}
$$

for all $i, j>n_{0}(\varepsilon)$. Using the definition of $g$ we obtain for each fixed $k \in \mathbb{N}$ that

$$
\left|\left(B^{r, s} x^{i}\right)_{k}-\left(B^{r, s} x^{j}\right)_{k}\right|^{p_{k} / L} \leq \sup _{k \in \mathbb{N}}\left|\left(B^{r, s} x^{i}\right)_{k}-\left(B^{r, s} x^{j}\right)_{k}\right|^{p_{k} / L}<\frac{\varepsilon}{2}
$$


for every $i, j>n_{0}(\varepsilon)$ which leads to the fact that $\left\{\left(B^{r, s} x^{0}\right)_{k},\left(B^{r, s} x^{1}\right)_{k},\left(B^{r, s} x^{2}\right)_{k}, \ldots\right\}$ is a Cauchy sequence of real numbers for every fixed $k \in \mathbb{N}$. Since $\mathbb{R}$ is complete, it converges, say $\left(B^{r, s} x^{i}\right)_{k} \rightarrow\left(B^{r, s} x\right)_{k}$ as $i \rightarrow \infty$. Using these infinitely many limits $\left(B^{r, s} x\right)_{0},\left(B^{r, s} x\right)_{1}, \ldots$, we define the sequence $\left\{\left(B^{r, s} x\right)_{0},\left(B^{r, s} x\right)_{1}, \ldots\right\}$. From (2.8) with $j \rightarrow \infty$, we have

$$
\left|\left(B^{r, s} x^{i}\right)_{k}-\left(B^{r, s} x\right)_{k}\right|^{p_{k} / L} \leq \frac{\varepsilon}{2}\left(i, j>n_{0}(\varepsilon)\right)
$$

for every fixed $k \in \mathbb{N}$. Since $x^{i}=\left\{x_{k}^{(i)}\right\} \in b_{0}^{r, s}(p)$ for each $i \in \mathbb{N}$, there exists $k_{0}(\varepsilon) \in \mathbb{N}$ such that

$$
\left|\left(B^{r, s} x^{i}\right)_{k}\right|^{p_{k} / L}<\frac{\varepsilon}{2}
$$

for every $k \geq k_{0}(\varepsilon)$ and for each fixed $i \in \mathbb{N}$. Therefore, taking a fixed $i>n_{0}(\varepsilon)$ we obtain by (2.9) and (2.10) that

$$
\left|\left(B^{r, s} x\right)_{k}\right|^{p_{k} / L} \leq\left|\left(B^{r, s} x\right)_{k}-\left(B^{r, s} x^{i}\right)_{k}\right|^{p_{k} / L}+\left|\left(B^{r, s} x^{i}\right)_{k}\right|^{p_{k} / L}<\frac{\varepsilon}{2}
$$

for every $k>k_{0}(\varepsilon)$. This shows that $x \in b_{0}^{r, s}(p)$. Since $\left\{x^{i}\right\}$ was an arbitrary Cauchy sequence, the space $b_{0}^{r, s}(p)$ is complete and this concludes the proof.

Now lets show that, $b^{r, s}(p)$ is the complete linear metric space paranormed by $h$ defined by (2.4). It is easy to see that the space $b^{r, s}(p)$ is linear with respect to the coordinate-wise addition and scalar multiplication. Therefore, we first show that it is a paranormed space with the paranorm $h$ defined by (2.4).

It is clear that $h(\theta)=0$ where $\theta=(0,0,0, \ldots)$ and $h(x)=h(-x)$ for all $x \in b^{r, s}(p)$.

Let $x, y \in b^{r, s}(p)$; then by Minkowski's inequality we have

$$
\begin{aligned}
h(x+y) & =\left(\sum_{k=0}^{\infty}\left|\frac{1}{(s+r)^{k}} \sum_{j=0}^{k}\left(\begin{array}{c}
k \\
j
\end{array}\right) s^{k-j} r^{j}\left(x_{j}+y_{j}\right)\right|^{p_{k}}\right)^{1 / M} \\
& =\left(\sum_{k=0}^{\infty}\left[\left|\frac{1}{(s+r)^{k}} \sum_{j=0}^{k}\left(\begin{array}{c}
k \\
j
\end{array}\right) s^{k-j} r^{j}\left(x_{j}+y_{j}\right)\right|^{p_{k} / M}\right]^{M}\right)^{1 / M} \\
& \leq\left(\sum_{k=0}^{\infty}\left|\frac{1}{(s+r)^{k}} \sum_{j=0}^{k}\left(\begin{array}{c}
k \\
j
\end{array}\right) s^{k-j} r^{j} x_{j}\right|^{p_{k}}\right)^{1 / M}+\left(\sum_{k=0}^{\infty}\left[\left.\frac{1}{(s+r)^{k}} \sum_{j=0}^{k}\left(\begin{array}{c}
k \\
j
\end{array}\right) s^{k-j} r^{j} y_{j}\right|^{p_{k}}\right)^{1 / M}\right. \\
& =h(x)+h(y)
\end{aligned}
$$

and for any $\alpha \in \mathbb{R}$ we immediately see that

$$
|\alpha|^{p_{k}} \leq \max \left\{1,|\alpha|^{M}\right\}
$$

Let $\left\{x^{n}\right\}$ be any sequence of the points $x^{n} \in b^{r, s}(p)$ such that $h\left(x^{n}-x\right) \rightarrow 0$ and $\left(\lambda_{n}\right)$ also be any sequence of scalars such that $\lambda_{n} \rightarrow \lambda$. We observe that

$$
h\left(\lambda_{n} x^{n}-\lambda x\right) \leq h\left[\left(\lambda_{n}-\lambda\right)\left(x^{n}-x\right)\right]+h\left[\lambda\left(x^{n}-x\right)\right]+h\left[\left(\lambda_{n}-\lambda\right) x\right] .
$$

It follows from $\lambda_{n} \rightarrow \lambda(n \rightarrow \infty)$ that $\left|\lambda_{n}-\lambda\right|<1$ for all sufficiently large $n$; hence

$$
\lim _{n \rightarrow \infty} h\left[\left(\lambda_{n}-\lambda\right)\left(x^{n}-x\right)\right] \leq \lim _{n \rightarrow \infty} h\left(x^{n}-x\right)=0 .
$$

Furthermore, we have

$$
\lim _{n \rightarrow \infty} h\left[\lambda\left(x^{n}-x\right)\right] \leq \max \left\{1,|\lambda|^{M}\right\} \lim _{n \rightarrow \infty} h\left(x^{n}-x\right)=0 .
$$

Also, we have

$$
\left.\lim _{n \rightarrow \infty} h\left[\left(\lambda_{n}-\lambda\right) x\right)\right] \leq \lim _{n \rightarrow \infty}\left|\lambda_{n}-\lambda\right| h(x)=0
$$

Then, we obtain from (2.13), (2.14), (2.15) and (2.16) that $h\left(\lambda_{n} x^{n}-\lambda x\right) \rightarrow 0$, as $n \rightarrow \infty$. This shows that $h$ is a paranorm on $b^{r, s}(p)$. Now, we show that $b^{r, s}(p)$ is complete. Let $\left\{x^{n}\right\}$ be any Cauchy sequence in the space $b^{r, s}(p)$, where $x^{n}=\left\{x_{0}^{(n)}, x_{1}^{(n)}, x_{2}^{(n)}, \ldots\right\}$. Then, for a given $\varepsilon>0$, there exists a positive integer $n_{0}(\varepsilon)$ such that $h\left(x^{n}-x^{m}\right)<\varepsilon$ for all $n, m>n_{0}(\varepsilon)$. Since for each fixed $k \in \mathbb{N}$ that

$$
\left|\left(B^{r, s} x^{n}\right)_{k}-\left(B^{r, s} x^{m}\right)_{k}\right| \leq\left[\sum_{k}\left|\left(B^{r, s} x^{n}\right)_{k}-\left(B^{r, s} x^{m}\right)_{k}\right|^{p_{k}}\right]^{\frac{1}{M}}=h\left(x^{n}-x^{m}\right)<\varepsilon
$$

for every $n, m>n_{0}(\varepsilon),\left\{\left(B^{r, s} x^{0}\right)_{k},\left(B^{r, s} x^{1}\right)_{k},\left(B^{r, s} x^{2}\right)_{k}, \ldots\right\}$ is a Cauchy sequence of real numbers for every fixed $k \in \mathbb{N}$. Since $\mathbb{R}$ is complete, it converges, say $\left(B^{r, s} x^{n}\right)_{k} \rightarrow\left(B^{r, s} x\right)_{k}$ as $n \rightarrow \infty$. Using these infinitely many limits $\left(B^{r, s} x\right)_{0},\left(B^{r, s} x\right)_{1}, \ldots$, we define the sequence $\left\{\left(B^{r, s} x\right)_{0},\left(B^{r, s} x\right)_{1}, \ldots\right\}$. For each $K \in \mathbb{N}$ and $n, m>n_{0}(\varepsilon)$

$$
\left[\sum_{k=0}^{K}\left|\left(B^{r, s} x^{n}\right)_{k}-\left(B^{r, s} x^{m}\right)_{k}\right|^{p_{k}}\right]^{\frac{1}{M}} \leq h\left(x^{n}-x^{m}\right)<\varepsilon .
$$


By letting $m, K \rightarrow \infty$, we have for $n>n_{0}(\varepsilon)$ that

$$
h\left(x^{n}-x\right)=\left[\sum_{k}\left|\left(B^{r, s} x^{n}\right)_{k}-\left(B^{r, s} x\right)_{k}\right|^{p_{k}}\right]^{\frac{1}{M}}<\varepsilon .
$$

This shows that $x^{n}-x \in b^{r, s}(p)$. Since $b^{r, s}(p)$ is a linear space, we conclude that $x \in b^{r, s}(p)$; it follows that $x^{n} \rightarrow x$, as $n \rightarrow \infty$ in $b^{r, s}(p)$, thus we have shown that $b^{r, s}(p)$ is complete.

Note that the absolute property does not hold on the spaces $b_{0}^{r, s}(p), b_{c}^{r, s}(p)$ and $b^{r, s}(p)$, since there exists at least one sequence in the spaces $b_{0}^{r, s}(p), b_{c}^{r, s}(p)$ and $b^{r, s}(p)$ and such that $g(x) \neq g(|x|)$, where $|x|=\left(\left|x_{k}\right|\right)$. This says that $b_{0}^{r, s}(p), b_{c}^{r, s}(p)$ and $b^{r, s}(p)$ are the sequence spaces of non-absolute type.

Theorem 2.2. The sequence spaces $b_{0}^{r, s}(p), b_{c}^{r, s}(p), b_{\infty}^{r, s}(p)$ and $b^{r, s}(p)$ are linearly isomorphic to the spaces $c_{0}(p)$, $c(p)$, $\ell_{\infty}(p)$ and $\ell(p)$, respectively, where $0<p_{k} \leq H<\infty$.

Proof. To avoid repetition of similar statements, we give the proof only for $b_{0}^{r, s}(p)$. We should show the existence of a linear bijection between the spaces $b_{0}^{r, s}(p)$ and $c_{0}(p)$. With the notation of (2.2), define the transformation $T$ from $b_{0}^{r, s}(p)$ to $c_{0}(p)$ by $x \mapsto y=T x$. The linearity of $T$ is trivial. Furthermore, it is obvious that $x=\theta$ whenever $T x=\theta$, and hence $T$ is injective.

Let $y \in c_{0}(p)$ and define the sequence

$$
x_{k}=\frac{1}{r^{k}} \sum_{j=0}^{k}\left(\begin{array}{l}
k \\
j
\end{array}\right)(-s)^{k-j}(s+r)^{j} y_{j} ; \quad(k \in \mathbb{N}) \text {. }
$$

Then, we have

$$
\begin{aligned}
& \left(B^{r, s} x\right)_{n}=\frac{1}{(s+r)^{n}} \sum_{k=0}^{n}\left(\begin{array}{l}
n \\
k
\end{array}\right) s^{n-k} r^{k} x_{k} \\
& =\frac{1}{(s+r)^{n}} \sum_{k=0}^{n}\left(\begin{array}{l}
n \\
k
\end{array}\right) s^{n-k} \sum_{j=0}^{k}\left(\begin{array}{l}
k \\
j
\end{array}\right)(-s)^{k-j}(s+r)^{j} y_{j} \\
& =\frac{1}{(s+r)^{n}} \sum_{j=0}^{n}\left(\sum_{k=j}^{n}\left(\begin{array}{l}
n \\
k
\end{array}\right)\left(\begin{array}{l}
k \\
j
\end{array}\right) s^{n-k}(-s)^{k-j}(s+r)^{j}\right) y_{j} \\
& =\frac{1}{(s+r)^{n}} \sum_{j=0}^{n}\left(\sum_{k=j}^{n}\left(\begin{array}{l}
n \\
j
\end{array}\right)\left(\begin{array}{l}
n-j \\
k-j
\end{array}\right)(-1)^{k-j} s^{n-j}(s+r)^{j}\right) y_{j} \\
& =\frac{1}{(s+r)^{n}} \sum_{j=0}^{n}\left(\begin{array}{l}
n \\
j
\end{array}\right) s^{n-j}(s+r)^{j}\left(\sum_{k=j}^{n}\left(\begin{array}{l}
n-j \\
k-j
\end{array}\right)(-1)^{k-j}\right) y_{j} \\
& =\frac{1}{(s+r)^{n}} \sum_{j=0}^{n}\left(\begin{array}{l}
n \\
j
\end{array}\right) s^{n-j}(s+r)^{j} \delta_{n k} y_{j} \\
& =\frac{1}{(s+r)^{n}}\left(\begin{array}{l}
n \\
n
\end{array}\right) s^{n-n}(s+r)^{n} 1 y_{n} \\
& =y_{n} \text {. }
\end{aligned}
$$

Thus, we have that $x \in b_{0}^{r, s}(p)$ and consequently $T$ is surjective. Hence, $T$ is a linear bijection and this says that the spaces $b_{0}^{r, s}(p)$ and $c_{0}(p)$ are linearly isomorphic, as was desired.

\section{The basis for the spaces $b_{0}^{r, s}(p), b_{c}^{r, s}(p)$ and $b^{r, s}(p)$}

Let $(\lambda, g)$ be a paranormed space. Recall that a sequence $\left(\beta_{k}\right)$ of the elements of $\lambda$ is called a basis for $\lambda$ if and only if, for each $x \in \lambda$, there exists a unique sequence $\left(\alpha_{k}\right)$ of scalars such that

$$
g\left(x-\sum_{k=0}^{n} \alpha_{k} \beta_{k}\right) \rightarrow 0 \text { as } n \rightarrow \infty .
$$

The series $\sum \alpha_{k} \beta_{k}$ which has the sum $x$ is then called the expansion of $x$ with respect to $\left(\beta_{n}\right)$, and written as $x=\sum \alpha_{k} \beta_{k}$. Since it is known that the matrix domain $\lambda_{A}$ of a sequence space $\lambda$ has a basis if and only if $\lambda$ has a basis whenever $A=\left(a_{n k}\right)$ is a triangle (cf. [11, Remark 2.4]), we have the following. Because of the isomorphism $T$ is onto, defined in the proof of Theorem 2.2, the inverse image of the basis of those spaces $c_{0}(p), c(p)$ and $\ell(p)$ are the basis of the new spaces $b_{0}^{r, s}(p), b_{c}^{r, s}(p)$ and $b^{r, s}(p)$, respectively. Therefore, we have the following:

Theorem 3.1. Let $\lambda_{k}=\left(B^{r, s} x\right)_{k}$ for all $k \in \mathbb{N}$ and $0<p_{k} \leq H<\infty$. Define the sequence $b^{(k)}=\left\{b^{(k)}\right\}_{k \in \mathbb{N}}$ of the elements of the space $b_{0}^{r, s}(p), b_{c}^{r, s}(p)$ and $b^{r, s}(p)$ by

$$
b_{n}^{(k)}=\left\{\begin{array}{cl}
\frac{1}{r^{n}}\left(\begin{array}{l}
n \\
k
\end{array}\right)(-s)^{n-k}(s+r)^{k} & , \quad n \geq k \\
0 & , \quad 0 \leq k<n
\end{array}\right.
$$

for every fixed $k \in \mathbb{N}$. Then 
(a) The sequence $\left\{b^{(k)}\right\}_{k \in \mathbb{N}}$ is a basis for the space $b_{0}^{r, s}(p)$, and any $x \in b_{0}^{r, s}(p)$ has a unique representation of the form

$$
x=\sum_{k} \lambda_{k} b^{(k)}
$$

(b) The set $\left\{e, b^{(1)}(r), b^{(2)}(r), \ldots\right\}$ is a basis for the space $b_{c}^{r, s}(p)$, and any $x \in b_{c}^{r, s}(p)$ has a unique representation of the form

$$
x=l e+\sum_{k}\left[\lambda_{k}-l\right] b^{(k)},
$$

where $l=\lim _{k \rightarrow \infty}\left(B^{r, s} x\right)_{k}$.

(c) The sequence $\left\{b^{(k)}\right\}_{k \in \mathbb{N}}$ is a basis for the space $b^{r, s}(p)$, and any $x \in b^{r, s}(p)$ has a unique representation of the form

$$
x=\sum_{k} \lambda_{k} b^{(k)} .
$$

\section{The $\alpha-, \beta-$ and $\gamma-$ duals of the spaces $b_{0}^{r, s}(p), b_{c}^{r, s}(p)$ and $b^{r, s}(p)$}

In this section, we state and prove the theorems determining the $\alpha-, \beta-$ and $\gamma$-duals of the sequence spaces $b_{0}^{r, s}(p), b_{c}^{r, s}(p)$ and $b^{r, s}(p)$ of non-absolute type.

We shall firstly give the definition of $\alpha-, \beta$ - and $\gamma$-duals of sequence spaces and after quoting the lemmas which are needed in proving the theorems given in Section 4 .

The set $S(\lambda, \mu)$ defined by

$$
S(\lambda, \mu)=\left\{z=\left(z_{k}\right) \in w: x z=\left(x_{k} z_{k}\right) \in \mu \text { for all } x=\left(x_{k}\right) \in \lambda\right\}
$$

is called the multiplier space of the sequence spaces $\lambda$ and $\mu$. One can eaisly observe for a sequence space $v$ with $\lambda \supset v \supset \mu$ that the inclusions

$$
S(\lambda, \mu) \subset S(v, \mu) \text { and } S(\lambda, \mu) \subset S(\lambda, v)
$$

hold. With the notation of (4.1), the alpha-, beta- and gamma-duals of a sequence space $\lambda$, which are respectively denoted by $\lambda^{\alpha}, \lambda^{\beta}$ and $\lambda^{\gamma}$ are defined by

$$
\lambda^{\alpha}=S\left(\lambda, \ell_{1}\right), \lambda^{\beta}=S(\lambda, c s) \text { and } \lambda^{\gamma}=S(\lambda, b s)
$$

The alpha-, beta- and gamma-duals of a sequence space are also referred as Köthe- Toeplitz dual, generalized Köthe-Toeplitz dual and Garling dual of a sequence space, respectively.

For to give the alpha-, beta- and gamma-duals of the spaces $b_{0}^{r, s}(p), b_{c}^{r, s}(p)$ and $b^{r, s}(p)$ of non-absolute type, we need the following lemma:

Lemma 4.1. $\left[10, q_{n}=1\right]$ Let $A=\left(a_{n k}\right)$ be an infinite matrix. Then, the following statements hold

(i) $A \in\left(c_{o}(p): \ell(q)\right)$ if and only if

$$
\sup _{K \in \mathscr{F}} \sum_{n}\left|\sum_{k \in K} a_{n k} M^{-1 / p_{k}}\right|<\infty, \quad \exists M \in \mathbb{N}_{2} .
$$

(ii) $A \in(c(p): \ell(q))$ if and only if (4.2) holds and

$$
\sum_{n}\left|\sum_{k} a_{n k}\right|<\infty .
$$

(iii) $A \in\left(c_{0}(p): c(q)\right)$ if and only if

$$
\begin{aligned}
& \sup _{n \in \mathbb{N}} \sum_{k}\left|a_{n k}\right| M^{-1 / p_{k}}<\infty, \exists M \in \mathbb{N}_{2}, \\
& \exists\left(\alpha_{k}\right) \subset \mathbb{R} \ni \lim _{n \rightarrow \infty}\left|a_{n k}-\alpha_{k}\right|=0 \text { for all } k \in \mathbb{N}, \\
& \exists\left(\alpha_{k}\right) \subset \mathbb{R} \ni \sup _{n \in \mathbb{N}} \sum_{k}\left|a_{n k}-\alpha_{k}\right| M^{-1 / p_{k}}<\infty, \exists M \in \mathbb{N}_{2} .
\end{aligned}
$$

(iv) $A \in(c(p): c(q))$ if and only if (4.4), (4.5), (4.6) hold and

$$
\exists \alpha \in \mathbb{R} \ni \lim _{n \rightarrow \infty}\left|\sum_{k} a_{n k}-\alpha\right|=0 .
$$


(v) $A \in\left(c_{o}(p): \ell_{\infty}(q)\right)$ if and only if

$$
\sup _{n \in \mathbb{N}} \sum_{k}\left|a_{n k}\right| M^{-1 / p_{k}}<\infty, \exists M \in \mathbb{N}_{2} .
$$

(vi) $A \in\left(c(p): \ell_{\infty}(q)\right)$ if and only if (4.8) holds and

$$
\sup _{n}\left|\sum_{k} a_{n k}\right|<\infty, \exists M \in \mathbb{N}_{2} .
$$

(vii) $A \in\left(\ell(p): \ell_{1}\right)$ if and only if

(a) Let $0<p_{k} \leq 1$ for all $k \in \mathbb{N}$. Then

$$
\sup _{N \in \mathscr{F}} \sup _{k \in \mathbb{N}}\left|\sum_{n \in N} a_{n k}\right|^{p_{k}}<\infty \text {. }
$$

(b) Let $1<p_{k} \leq H<\infty$ for all $k \in \mathbb{N}$. Then, there exists an integer $M>1$ such that

$$
\sup _{N \in \mathscr{F}} \sum_{k}\left|\sum_{n \in N} a_{n k} M^{-1}\right|^{p_{k}^{\prime}}<\infty .
$$

Lemma 4.2. [17] Let $A=\left(a_{n k}\right)$ be an infinite matrix. Then, the following statements hold (i) $A \in\left(\ell(p): \ell_{\infty}\right)$ if and only if

(a) Let $0<p_{k} \leq 1$ for all $k \in \mathbb{N}$. Then,

$$
\sup _{n, k \in \mathbb{N}}\left|a_{n k}\right|^{p_{k}}<\infty .
$$

(b) Let $1<p_{k} \leq H<\infty$ for all $k \in \mathbb{N}$. Then, there exists an integer $M>1$ such that

$$
\sup _{n \in \mathbb{N}} \sum_{k}\left|a_{n k} M^{-1}\right|^{p_{k}^{\prime}}<\infty .
$$

(ii) Let $0<p_{k} \leq H<\infty$ for all $k \in \mathbb{N}$. Then, $A=\left(a_{n k}\right) \in(\ell(p): c)$ if and only if (4.12) and (4.13) hold, and

$$
\lim _{n \rightarrow \infty} a_{n k}=\beta_{k}, \forall k \in \mathbb{N} \text {. }
$$

Theorem 4.3. Let $K \in \mathscr{F}$ and $K^{*}=\{k \in \mathbb{N}: n \geq k\} \cap K$ for $K \in \mathscr{F}$. Define the sets $T_{1}^{r}(p), T_{2}^{r}, T_{3}(p)$ and $T_{4}(p)$ as follows:

$$
\begin{aligned}
& T_{1}(p)=\bigcup_{M>1}\left\{a=\left(a_{k}\right) \in w: \sup _{K \in \mathscr{F}} \sum_{n}\left|\sum_{k \in K^{*}} c_{n k} M^{-1 / p_{k}}\right|<\infty\right\}, \\
& T_{2}=\left\{a=\left(a_{k}\right) \in w: \sum_{n}\left|\sum_{k=0}^{n} c_{n k}\right| \text { exists for each } n \in \mathbb{N}\right\}, \\
& T_{3}(p)=\bigcup_{M>1}\left\{a=\left(a_{k}\right) \in w: \sup _{N \in \mathscr{F}} \sum_{k}\left|\sum_{n \in N} c_{n k} M^{-1}\right|^{p_{k}^{\prime}}<\infty,\right\}, \\
& T_{4}(p)=\left\{a=\left(a_{k}\right) \in w: \sup _{N \in \mathscr{F}} \sup _{k \in \mathbb{N}}\left|\sum_{n \in N} c_{n k}\right|^{p_{k}}<\infty\right\},
\end{aligned}
$$

where the matrix $C=\left(c_{n k}\right)$ defined by

$$
c_{n k}=\left\{\begin{array}{cl}
\frac{1}{r^{n}} \sum_{k=0}^{n}\left(\begin{array}{l}
n \\
k
\end{array}\right)(-s)^{n-k}(s+r)^{k} a_{n} & , \quad 0 \leq k \leq n, \\
0 & , \quad k \geq n .
\end{array}\right.
$$

Then, $\left[b_{0}^{r, s}(p)\right]^{\alpha}=T_{1}(p),\left[b_{c}^{r, s}(p)\right]^{\alpha}=T_{1}(p) \cap T_{2}$ and

$$
\left[b^{r, s}(p)\right]^{\alpha}= \begin{cases}T_{3}(p) & 1<p_{k} \leq H<\infty, \forall k \in \mathbb{N} \\ T_{4}(p) & 0<p_{k} \leq 1, \forall k \in \mathbb{N}\end{cases}
$$

Proof. We chose the sequence $a=\left(a_{k}\right) \in w$. We can easily derive that with the (2.2) that

$$
a_{n} x_{n}=\frac{1}{r^{n}} \sum_{k=0}^{n}\left(\begin{array}{l}
n \\
k
\end{array}\right)(-s)^{n-k}(s+r)^{k} a_{n} y_{k}=(C y)_{n}, \quad(n \in \mathbb{N})
$$

for all $k, n \in \mathbb{N}$, where $C=\left(c_{n k}\right)$ defined by (4.15). It follows from (4.17) that $a x=\left(a_{n} x_{n}\right) \in \ell_{1}$ whenever $x \in b_{0}^{r, s}(p)$ if and only if $C y \in \ell_{1}$ whenever $y \in c_{0}(p)$. This means that $a=\left(a_{n}\right) \in\left[b_{0}^{r, s}(p)\right]^{\alpha}$ if and only if $C \in\left(c_{0}(p): \ell_{1}\right)$. Then, we derive by (4.2) with $q_{n}=1$ for all $n \in \mathbb{N}$ that $\left[b_{0}^{r, s}(p)\right]^{\alpha}=T_{1}^{r}(p)$.

Using the (4.3) with $q_{n}=1$ for all $n \in \mathbb{N}$ and (4.17), the proof of the $\left[b_{c}^{r, s}(p)\right]^{\alpha}=T_{1}^{r}(p) \cap T_{2}$ can also be obtained in a similar way. Also, using the (4.10),(4.11) and (4.17), the proof of the

$$
\left[b^{r, s}(p)\right]^{\alpha}= \begin{cases}T_{3}(p) & 1<p_{k} \leq H<\infty, \forall k \in \mathbb{N}, \\ T_{4}(p) & 0<p_{k} \leq 1, \forall k \in \mathbb{N},\end{cases}
$$

can also be obtained in a similar way. 
Theorem 4.4. The matrix $D=\left(d_{n k}\right)$ is defined by

$$
d_{n k}=\left\{\begin{array}{cll}
\sum_{j=k}^{n}\left(\begin{array}{l}
j \\
k
\end{array}\right)(-s)^{j-k} r^{-j}(r+s)^{k} a_{j} & , & (0 \leq k \leq n) \\
0 & , & (k>n)
\end{array}\right.
$$

for all $k, n \in \mathbb{N}$. Define the sets $T_{5}(p), T_{6}, T_{7}(p), T_{8}, T_{9}(p), T_{10}$ and $T_{11}(p)$ as follows:

$$
\begin{aligned}
& T_{5}(p)=\bigcup_{M>1}\left\{a=\left(a_{k}\right) \in w: \sup _{n \in \mathbb{N}} \sum_{k=0}^{n}\left|d_{n k}\right| M^{-1 / p_{k}}<\infty\right\}, \\
& T_{6}=\left\{a=\left(a_{k}\right) \in w: \lim _{n \rightarrow \infty}\left|d_{n k}\right| \text { exists for each } k \in \mathbb{N}\right\}, \\
& T_{7}(p)=\bigcup_{M>1}\left\{a=\left(a_{k}\right) \in w: \exists\left(\alpha_{k}\right) \subset \mathbb{R} \ni \sup _{n \in \mathbb{N}} \sum_{k=0}^{n}\left|d_{n k}-\alpha_{k}\right| M^{-1 / p_{k}}<\infty\right\}, \\
& T_{8}=\left\{a=\left(a_{k}\right) \in w: \lim _{n \rightarrow \infty} \sum_{k=0}^{n}\left|d_{n k}\right| \text { exists }\right\}, \\
& T_{9}(p)=\bigcup_{M>1}\left\{a=\left(a_{k}\right) \in w: \sup _{n \in \mathbb{N}} \sum_{k}\left|d_{n k} M^{-1}\right| p_{k}^{\prime}<\infty\right\}, \\
& T_{10}=\left\{a=\left(a_{k}\right) \in w: \lim _{n \rightarrow \infty} d_{n k} \text { exists for each } k \in \mathbb{N}\right\}, \\
& T_{11}(p)=\left\{a=\left(a_{k}\right) \in w: \sup _{n, k \in \mathbb{N}}\left|d_{n k}\right|^{p_{k}}<\infty\right\} .
\end{aligned}
$$

Then, $\left[b_{0}^{r, s}(p)\right]^{\beta}=T_{5}(p) \cap T_{6} \cap T_{7}(p),\left[b_{c}^{r, s}(p)\right]^{\beta}=\left[b_{0}^{r, s}(p)\right]^{\beta} \cap T_{8}$ and

$$
\left[b^{r, s}(p)\right]^{\beta}= \begin{cases}T_{9}(p) \cap T_{10} & , \quad 1<p_{k} \leq H<\infty, \forall k \in \mathbb{N} \\ T_{10} \cap T_{11}(p) & , \quad 0<p_{k} \leq 1, \forall k \in \mathbb{N}\end{cases}
$$

Proof. We give the proof again only for the space $b_{0}^{r, s}(p)$. Consider the equation

$$
\begin{aligned}
\sum_{k=0}^{n} a_{k} x_{k} & =\sum_{k=0}^{n}\left[\frac{1}{r^{k}} \sum_{j=0}^{k}\left(\begin{array}{l}
k \\
j
\end{array}\right)(-s)^{k-j}(s+r)^{j} y_{j}\right] a_{k} \\
& =\sum_{k=0}^{n}\left[\sum_{j=k}^{n}\left(\begin{array}{l}
j \\
k
\end{array}\right)(-s)^{j-k} r^{-j}(r+s)^{k} a_{j}\right] y_{k}=(D y)_{n},
\end{aligned}
$$

where $D=\left(d_{n k}\right)$ defined by (4.18). Thus, we deduce from (4.20) that $a x=\left(a_{k} x_{k}\right) \in c s$ whenever $x=\left(x_{k}\right) \in b_{0}^{r, s}(p)$ if and only if $D y \in c$ whenever $y=\left(y_{k}\right) \in c_{0}(p)$. That is to say that $a=\left(a_{k}\right) \in\left[b_{0}^{r, s}(p)\right]^{\beta}$ if and only if $D \in\left(c_{0}(p): c\right)$. Therefore, we derive from (4.4),(4.5) and (4.6) with $q_{n}=1$ for all $n \in \mathbb{N}$ that $\left[b_{0}^{r, s}(p)\right]^{\beta}=T_{5}(p) \cap T_{6} \cap T_{7}(p)$.

Using the (4.4),(4.5), (4.6) and (4.7) with $q_{n}=1$ for all $n \in \mathbb{N}$ and (4.20), the proofs of the $\left[b_{c}^{r, s}(p)\right]^{\beta}=\left[b_{0}^{r, s}(p)\right]^{\beta} \cap T_{8}$ can also be obtained in a similar way. Also, using the (4.12),(4.13), (4.14) and (4.20), the proofs of the

$$
\left[b^{r, s}(p)\right]^{\beta}= \begin{cases}T_{9}(p) \cap T_{10} & , \quad 1<p_{k} \leq H<\infty, \forall k \in \mathbb{N} \\ T_{10} \cap T_{11}(p) & , \quad 0<p_{k} \leq 1, \forall k \in \mathbb{N}\end{cases}
$$

can also be obtained in a similar way.

Theorem 4.5. Define the set $T_{12}$ by

$$
T_{12}=\left\{a=\left(a_{k}\right) \in w: \sup _{n}\left|\sum_{k} a_{n k}\right|<\infty\right\} .
$$

Then, $\left[b_{0}^{r, s}(p)\right]^{\gamma}=T_{5}(p),\left[b_{c}^{r, s}(p)\right]^{\gamma}=\left[b_{0}^{r, s}(p)\right]^{\gamma} \cap T_{12}$ and

$$
\left[b^{r, s}(p)\right]^{\gamma}= \begin{cases}T_{8}(p) \quad, \quad 1<p_{k} \leq H<\infty, \forall k \in \mathbb{N} \\ T_{10}(p) \quad, \quad 0<p_{k} \leq 1, \forall k \in \mathbb{N}\end{cases}
$$

Proof. This is obtained in the similar way used in the proof of Theorem 4.4. 


\section{Certain matrix mappings on the sequence spaces $b_{0}^{r, s}(p), b_{c}^{r, s}(p)$ and $b^{r, s}(p)$}

In this section, we characterize some matrix mappings on the spaces $b_{0}^{r, s}(p), b_{c}^{r, s}(p)$ and $b^{r, s}(p)$.

We known that, if $b_{0}^{r, s}(p) \cong c_{0}(p), b_{c}^{r, s}(p) \cong c(p)$ and $b^{r, s}(p) \cong \ell(p)$, we can say: The equivalence " $x \in b_{0}^{r, s}(p), b_{c}^{r, s}(p)$ or $b^{r, s}(p)$ if and only if $y \in c_{0}(p), c(p)$ or $\ell(p)$ " holds.

In what follows, for brevity, we write,

$$
\tilde{a}_{n k}:=\sum_{j=k}^{n}\left(\begin{array}{l}
j \\
k
\end{array}\right)(-s)^{j-k} r^{-j}(r+s)^{k} a_{n j}
$$

for all $k, n \in \mathbb{N}$.

Theorem 5.1. Suppose that the entries of the infinite matrices $A=\left(a_{n k}\right)$ and $E=\left(e_{n k}\right)$ are connected with the relation

$$
e_{n k}:=\tilde{a}_{n k}
$$

for all $k, n \in \mathbb{N}$ and $\mu$ be any given sequence space. Then,

(i) $A \in\left(b_{0}^{r, s}(p): \mu\right)$ if and only if $\left\{a_{n k}\right\}_{k \in \mathbb{N}} \in\left\{b_{0}^{r, s}(p)\right\}^{\beta}$ for all $n \in \mathbb{N}$ and $E \in\left(c_{0}(p): \mu\right)$.

(ii) $A \in\left(b_{c}^{r, s}(p): \mu\right)$ if and only if $\left\{a_{n k}\right\}_{k \in \mathbb{N}} \in\left\{b_{c}^{r, s}(p)\right\}^{\beta}$ for all $n \in \mathbb{N}$ and $E \in(c(p): \mu)$.

(iii) $A \in\left(b^{r, s}(p): \mu\right)$ if and only if $\left\{a_{n k}\right\}_{k \in \mathbb{N}} \in\left\{b^{r, s}(p)\right\}^{\beta}$ for all $n \in \mathbb{N}$ and $E \in(\ell(p): \mu)$.

Proof. We prove only part of (i). Let $\mu$ be any given sequence space. Suppose that (5.1) holds between $A=\left(a_{n k}\right)$ and $E=\left(e_{n k}\right)$, and take into account that the spaces $b_{0}^{r, s}(p)$ and $c_{0}(p)$ are linearly isomorphic.

Let $A \in\left(b_{0}^{r, s}(p): \mu\right)$ and take any $y=\left(y_{k}\right) \in c_{0}(p)$. Then $E B^{r, s}$ exists and $\left\{a_{n k}\right\}_{k \in \mathbb{N}} \in T_{5}(p) \cap T_{6}$ which yields that $\left\{e_{n k}\right\}_{k \in \mathbb{N}} \in c_{0}(p)$ for each $n \in \mathbb{N}$. Hence, Ey exists and thus

$$
\sum_{k} e_{n k} y_{k}=\sum_{k} a_{n k} x_{k}
$$

for all $n \in \mathbb{N}$.

We have that $E y=A x$ which leads us to the consequence $E \in\left(c_{0}(p): \mu\right)$.

Conversely, let $\left\{a_{n k}\right\}_{k \in \mathbb{N}} \in\left\{b_{0}^{r, s}(p)\right\}^{\beta}$ for each $n \in \mathbb{N}$ and $E \in\left(c_{0}(p): \mu\right)$ hold, and take any $x=\left(x_{k}\right) \in b_{0}^{r, s}(p)$. Then, $A x$ exists. Therefore, we obtain from the equality

$$
\sum_{k=0}^{\infty} a_{n k} x_{k}=\sum_{k=0}^{\infty}\left[\sum_{j=0}^{k}\left(\begin{array}{l}
j \\
k
\end{array}\right)(-r)^{j-k}(1-r)^{-(j+1)} a_{n j}\right] y_{k}
$$

for all $n \in \mathbb{N}$, that $E y=A x$ and this shows that $A \in\left(b_{0}^{r, s}(p): \mu\right)$. This completes the proof of part of (i).

Theorem 5.2. Suppose that the elements of the infinite matrices $A=\left(a_{n k}\right)$ and $B=\left(b_{n k}\right)$ are connected with the relation

$$
b_{n k}:=\frac{1}{(s+r)^{n}} \sum_{j=0}^{n}\left(\begin{array}{c}
n \\
j
\end{array}\right) s^{n-j} r^{j} a_{j k} \text { for all } k, n \in \mathbb{N} .
$$

Let $\mu$ be any given sequence space. Then,

(i) $A \in\left(\mu: b_{0}^{r, s}(p)\right)$ if and only if $B \in\left(\mu: c_{0}(p)\right)$.

(ii) $A \in\left(\mu: b_{c}^{r, s}(p)\right)$ if and only if $B \in(\mu: c(p))$.

(iii) $A \in\left(\mu: b^{r, s}(p)\right)$ if and only if $B \in(\mu: \ell(p))$.

Proof. We prove only part of (i). Let $z=\left(z_{k}\right) \in \mu$ and consider the following equality.

$$
\sum_{k=0}^{m} b_{n k} z_{k}=\sum_{j=n}^{\infty}\left(\begin{array}{l}
j \\
n
\end{array}\right)(1-r)^{n+1} r^{j-n}\left(\sum_{k=0}^{m} a_{j k} z_{k}\right) \text { for all } m, n \in \mathbb{N}
$$

which yields as $m \rightarrow \infty$ that $(B z)_{n}=\left\{B^{r, s}(A z)\right\}_{n}$ for all $n \in \mathbb{N}$. Therefore, one can observe from here that $A z \in b_{0}^{r, s}(p)$ whenever $z \in \mu$ if and only if $B z \in c_{0}(p)$ whenever $z \in \mu$. This completes the proof of part of (i).

Of course, Theorems 5.1 and 5.2 have several consequences depending on the choice of the sequence space $\mu$. Whence by Theorem 5.1 and Theorem 5.2, the necessary and sufficient conditions for $\left(b_{0}^{r, s}(p): \mu\right),\left(\mu: b_{0}^{r, s}(p)\right),\left(b_{c}^{r, s}(p): \mu\right),\left(\mu: b_{c}^{r, s}(p)\right)$ and $\left(b^{r, s}(p): \mu\right),\left(\mu: b^{r, s}(p)\right)$ may be derived by replacing the entries of $C$ and $A$ by those of the entries of $E=C\left\{B^{r, s}\right\}^{-1}$ and $B=B^{r, s} A$, respectively; where the necessary and sufficient conditions on the matrices $E$ and $B$ are read from the concerning results in the existing literature.

The necessary and sufficient conditions characterizing the matrix mappings between the sequence spaces of Maddox are determined by Grosse-Erdmann [10]. Let $N$ and $K$ denote the finite subset of $\mathbb{N}, L$ and $M$ also denote the natural numbers. Prior to giving the theorems, let us suppose that $\left(q_{n}\right)$ is a non-decreasing bounded sequence of positive numbers and consider the following conditions:

$$
\lim _{n}\left|a_{n k}\right|^{q_{n}}=0, \text { for all } k .
$$




$$
\begin{aligned}
& \forall L, \exists M \ni \sup _{n} L^{1 / q_{n}} \sum_{k}\left|a_{n k}\right| M^{-1 / p_{k}}<\infty, \\
& \lim _{n}\left|\sum_{k} a_{n k}\right|^{q_{n}}=0, \\
& \forall L, \sup _{n} \sup _{k \in K_{1}}\left|a_{n k} L^{1 / q_{n}}\right|^{p_{k}}<\infty, \\
& \forall L, \exists M \ni \sup _{n} \sum_{k \in K_{2}}\left|a_{n k} L^{1 / q_{n}} M^{-1}\right|^{p_{k}^{\prime}}<\infty, \\
& \forall M, \lim _{n}\left(\sum_{k}\left|a_{n k}\right| M^{1 / p_{k}}\right)^{q_{n}}=0, \\
& \forall M, \sup _{n} \sum_{k}\left|a_{n k}\right| M^{1 / p_{k}}<\infty, \\
& \forall M, \sup _{K} \sum_{n}\left|\sum_{k \in K} a_{n k} M^{1 / p_{k}}\right|^{q_{n}}<\infty .
\end{aligned}
$$

Lemma 5.3. Let $A=\left(a_{n k}\right)$ be an infinite matrix. Then

(i) $A=\left(a_{n k}\right) \in\left(c_{0}(p): \ell_{\infty}(q)\right)$ if and only if (4.8) holds.

(ii) $A=\left(a_{n k}\right) \in\left(c(p): \ell_{\infty}(q)\right)$ if and only if (4.8) and (4.9) hold.

(iii) $A=\left(a_{n k}\right) \in\left(\ell(p): \ell_{\infty}\right)$ if and only if (4.12) and (4.13) hold.

(iv) $A=\left(a_{n k}\right) \in\left(c_{0}(p): c(q)\right)$ if and only if (4.4), (4.5) and (4.6) hold.

(v) $A=\left(a_{n k}\right) \in(c(p): c(q))$ if and only if (4.4), (4.5), (4.6) and (4.7) hold.

(vi) $A=\left(a_{n k}\right) \in(\ell(p): c)$ if and only if (4.12), (4.13) and (4.14) hold.

(vii) $A=\left(a_{n k}\right) \in\left(c_{0}(p): c_{0}(q)\right)$ if and only if (5.3) and (5.4) hold.

(viii) $A=\left(a_{n k}\right) \in\left(c(p): c_{0}(q)\right)$ if and only if (5.3), (5.4) and (5.5) hold.

(ix) $A=\left(a_{n k}\right) \in\left(\ell(p): c_{0}(q)\right)$ if and only if (5.3), (5.6) and (5.7) hold.

(x) $A=\left(a_{n k}\right) \in\left(\ell_{\infty}(p): c_{0}(q)\right)$ if and only if (5.8) holds.

(xi) $A=\left(a_{n k}\right) \in\left(\ell_{\infty}(p): c(q)\right)$ if and only if (5.9) holds.

(xii) $A=\left(a_{n k}\right) \in\left(\ell_{\infty}(p): \ell(q)\right)$ if and only if (5.10) holds.

(xiii) $A=\left(a_{n k}\right) \in\left(c_{0}(p): \ell(q)\right)$ if and only if (4.2) holds.

(xiv) $A=\left(a_{n k}\right) \in(c(p): \ell(q))$ if and only if (4.2) and (4.4) hold.

Corollary 5.4. Let $A=\left(a_{n k}\right)$ be an infinite matrix. The following statements hold:

(i) $A \in\left(b_{0}^{r, s}(p): \ell_{\infty}(q)\right)$ if and only if $\left\{a_{n k}\right\}_{k \in \mathbb{N}} \in\left\{b_{0}^{r, s}(p)\right\}^{\beta}$ for all $n \in \mathbb{N}$ and (4.8) holds with $\tilde{a}_{n k}$ instead of $a_{n k}$ with $q=1$.

(ii) $A \in\left(b_{0}^{r, s}(p): c_{0}(q)\right)$ if and only if $\left\{a_{n k}\right\}_{k \in \mathbb{N}} \in\left\{b_{0}^{r, s}(p)\right\}^{\beta}$ for all $n \in \mathbb{N}$ and (5.3) and (5.4) hold with $\tilde{a}_{n k}$ instead of $a_{n k}$ with $q=1$.

(iii) $A \in\left(b_{0}^{r, s}(p): c(q)\right)$ if and only if $\left\{a_{n k}\right\}_{k \in \mathbb{N}} \in\left\{b_{0}^{r, s}(p)\right\}^{\beta}$ for all $n \in \mathbb{N}$ and (4.4), (4.5) and (4.6) hold with $\tilde{a}_{n k}$ instead of $a_{n k}$ with $q=1$.

Corollary 5.5. Let $A=\left(a_{n k}\right)$ be an infinite matrix. The following statements hold:

(i) $A \in\left(b_{c}^{r, s}(p): \ell_{\infty}(q)\right)$ if and only if $\left\{a_{n k}\right\}_{k \in \mathbb{N}} \in\left\{b_{c}^{r, s}(p)\right\}^{\beta}$ for all $n \in \mathbb{N}$ and (4.8) and (4.9) hold with $\tilde{a}_{n k}$ instead of $a_{n k}$ with $q=1$.

(ii) $A \in\left(b_{c}^{r, s}(p): c_{0}(q)\right)$ if and only if $\left\{a_{n k}\right\}_{k \in \mathbb{N}} \in\left\{b_{c}^{r, s}(p)\right\}^{\beta}$ for all $n \in \mathbb{N}$ and (5.3), (5.4) and (5.5) hold with $\tilde{a}_{n k}$ instead of $a_{n k}$ with $q=1$.

(iii) $A \in\left(b_{c}^{r, s}(p): c(q)\right)$ if and only if $\left\{a_{n k}\right\}_{k \in \mathbb{N}} \in\left\{b_{c}^{r, s}(p)\right\}^{\beta}$ for all $n \in \mathbb{N}$ and (4.4), (4.5), (4.6) and (4.7) hold with $\tilde{a}_{n k}$ instead of $a_{n k}$ with $q=1$.

Corollary 5.6. Let $A=\left(a_{n k}\right)$ be an infinite matrix. The following statements hold:

(i) $A \in\left(b^{r, s}(p): \ell_{\infty}\right)$ if and only if $\left\{a_{n k}\right\}_{k \in \mathbb{N}} \in\left\{b^{r, s}(p)\right\}^{\beta}$ for all $n \in \mathbb{N}$ and (4.12) and (4.13) hold with $\tilde{a}_{n k}$ instead of $a_{n k}$.

(ii) $A \in\left(b^{r, s}(p): c_{0}(q)\right)$ if and only if $\left\{a_{n k}\right\}_{k \in \mathbb{N}} \in\left\{b^{r, s}(p)\right\}^{\beta}$ for all $n \in \mathbb{N}$ and (5.3), (5.6) and (5.7) hold with $\tilde{a}_{n k}$ instead of $a_{n k}$ with $q=1$.

(iii) $A \in\left(b^{r, s}(p): c\right)$ if and only if $\left\{a_{n k}\right\}_{k \in \mathbb{N}} \in\left\{b^{r, s}(p)\right\}^{\beta}$ for all $n \in \mathbb{N}$ and (4.12), (4.13) and (4.14) hold with $\tilde{a}_{n k}$ instead of $a_{n k}$.

Corollary 5.7. Let $A=\left(a_{n k}\right)$ be an infinite matrix and $b_{n k}$ be defined by (5.2). Then, following statements hold:

(i) $A \in\left(\ell_{\infty}(q): b_{0}^{r, s}(p)\right)$ if and only if (5.8) holds with $b_{n k}$ instead of $a_{n k}$ with $q=1$.

(ii) $A \in\left(c_{0}(q): b_{0}^{r, s}(p)\right)$ if and only if (5.3) and (5.4) hold with $b_{n k}$ instead of $a_{n k}$ with $q=1$.

(iii) $A \in\left(c(q): b_{0}^{r, s}(p)\right)$ if and only if (5.3), (5.4) and (5.5) holds with $b_{n k}$ instead of $a_{n k}$ with $q=1$.

Corollary 5.8. Let $A=\left(a_{n k}\right)$ be an infinite matrix and $b_{n k}$ be defined by (5.2). Then, following statements hold:

(i) $A \in\left(\ell_{\infty}(q): b_{c}^{r, s}(p)\right)$ if and only if (5.9) holds with $b_{n k}$ instead of $a_{n k}$ with $q=1$.

(ii) $A \in\left(c_{0}(q): b_{c}^{r, s}(p)\right)$ if and only if (4.4), (4.5) and (4.6) hold with $b_{n k}$ instead of $a_{n k}$ with $q=1$.

(iii) $A \in\left(c(q): b_{c}^{r, s}(p)\right)$ if and only if (4.4), (4.5), (4.6) and (4.7) hold with $b_{n k}$ instead of $a_{n k}$ with $q=1$.

Corollary 5.9. Let $A=\left(a_{n k}\right)$ be an infinite matrix and $b_{n k}$ be defined by (5.2). Then, following statements hold:

(i) $A \in\left(\ell_{\infty}(q): b^{r, s}(p)\right)$ if and only if (5.10) holds with $b_{n k}$ instead of $a_{n k}$ with $q=1$.

(ii) $A \in\left(c_{0}(q): b^{r, s}(p)\right)$ if and only if (4.2) holds with $b_{n k}$ instead of $a_{n k}$ with $q=1$.

(iii) $A \in\left(c(q): b^{r, s}(p)\right)$ if and only if (4.2) and (4.4) hold with $b_{n k}$ instead of $a_{n k}$ with $q=1$. 


\section{References}

[1] B. Altay, F. Başar, On the paranormed Riesz sequence spaces of non-absolute type, Southeast Asian Bull. Math., 26, 701-715 (2002)

[2] B. Altay, F. Başar, Some paranormed Riesz sequence spaces of non-absolute type, Southeast Asian Bull. Math., 30, 591-608 (2006).

[3] F. Başar, B. Altay, Matrix mappings on the space $b s(p)$ and its $\alpha-, \beta-$ and $\gamma-$ duals, Aligarh Bull. Math., 21(1), 79-91 (2002).

[4] F. Başar, Infinite matrices and almost boundedness, Boll. Un. Mat. Ital., 6(7), 395-402 (1992).

[5] M. C. Bişgin, The binomial sequence spaces of nonabsolute type, J. Inequal. Appl. 309 (2016).

[6] M. C. Bişgin, The binomial sequence spaces which include the spaces $\ell_{p}$ and $\ell_{\infty}$ and geometric properties, J. Inequal. Appl. 304 (2016).

[7] B. Choudhary, S. K. Mishra, On Köthe-Toeplitz duals of certain sequence spaces and their matrix transformations, Indian J. Pure Appl. Math., 24(5), 291-301 (1993).

[8] S. Demiriz, C. Cakan, On Some New Paranormed Euler Sequence Spaces and Euler Core, Acta Math. Sin.(Eng. Ser.), 26(7), 1207-1222 (2010).

[9] S. Demiriz, H. B. Ellidokuzog̃lu, On The Paranormed Taylor Sequence Spaces, Konuralp Journal Of Mathematics, 4(2), 132-148 (2016).

[10] K. G. Grosse-Erdmann, Matrix transformations between the sequence spaces of Maddox. J. Math. Anal. Appl., 180, 223-238 (1993).

[11] A. Jarrah and E. Malkowsky, BK spaces, bases and linear operators, Rend. Circ. Mat. Palermo, 52(2), 177-191 (1990).

[12] E.E. Kara and M. İlkhan, On some Banach sequence spaces derived by a new band matrix, Br. J. Math. Comput. Sci., 9(2), 141-159 (2015).

[13] E.E. Kara and M. İlkhan, Some properties of generalized Fibonacci sequence spaces, Linear Multilinear Algebra, 64(11), 2208-2223 (2016).

[14] M. Kirişci, On the Taylor sequence spaces of nonabsulate type which include the spaces $c_{0}$ and $c, \mathrm{~J}$. Math. Anal., 6(2), 22-35 (2015).

[15] M. Kirişci, The application domain of infinite matrices with algorithms, Univ. J. Math. Appl., 1(1), 1-9 (2018).

[16] M. Candan and A. Güneş, Paranormed sequence space of non-absolute type founded using generalized difference matrix, Proc. Natl. Acad. Sci., India, Sect. A Phys. Sci. 85(2), 269-276 (2015).

[17] C. G. Lascarides and I. J. Maddox, Matrix transformations between some classes of sequences, Proc.Camb. Phil. Soc., 68, 99-104 (1970).

[18] I.J. Maddox, Elements of Functional Analysis, second ed., The University Press, Cambridge, 1988.

[19] I. J. Maddox, Paranormed sequence spaces generated by infinite matrices, Proc. Camb. Phios. Soc., 64, 335-340 (1968).

[20] H. Nakano, Modulared sequence spaces, Proc. Jpn. Acad., 27(2), 508-512 (1951).

[21] S. Simons, The sequence spaces $\ell\left(p_{v}\right)$ and $m\left(p_{v}\right)$. Proc. London Math. Soc., 15(3), 422-436 (1965). 